\title{
COMPONENTES DA VARIÂNCIA EM CARACTERES AGRONÔMICOS DE ACEROLA ${ }^{1}$
}

\author{
JOSÉ EVERALDO GOMES ${ }^{2}$, DILERMANDO PERECIN ${ }^{3}$ ANTÔNIO BALDO GERALDO MARTINS ${ }^{4}$
}

\begin{abstract}
RESUMO - Estimaram-se os componentes da variância e sua participação porcentual na variância de caracteres de genótipos de acerola de Itápolis, Viradouro e Jaboticabal. Realizaram-se avaliações em folhas, frutos e outros caracteres das plantas, de dez/97 a jan/ 99. Na estimação, adotaram-se os modelos com clones, épocas e clones x épocas, quando havia duas plantas do mesmo clone e com genótipos e épocas, quando havia uma planta por genótipo. Verificaram-se diferenças significativas em clones, épocas e clones x épocas em todos os caracteres, exceto comprimento de folhas (CMFO), rendimento de polpa (RMP20FR) e dias em colheita, que apresentaram significância em fatores específicos. A variância ambiental interfere mais acentuadamente em largura de folha (LMFO), massa de polpa, quantidade de vitamina C (VITC), RMP20FR e crescimento de ramos. Os proporcionalmente elevados componentes de variância genética de CMFO, LMFO, altura de planta, diâmetro de copa, altura de fruto, VITC e massa de fruto tornam efetiva a seleção de genótipos.
\end{abstract}

Termos para indexação: variância genotípica, variância de ambiente, Malpighia emarginata.

\section{VARIANCE COMPONENTS IN WEST INDIAN CHERRY AGRONOMIC CHARACTERS}

\begin{abstract}
The Variance components and their proportional participation in general variance of characters in West Indian cherry genotypes from Itápolis, Viradouro and Jaboticabal-SP-Brazil, were estimated. The Leaf, fruit and other plant characteres were evaluated from December/1997 to January/1999. The Models with clones, time and clones x time were used when there were two plants by clone; and with genotypes and time, when there was only one plant by genotype. Significant differences were verified in clones, time and clones $x$ time in all characteres, except in length of leaves (CMFO), pulp profit (RMF20FR) and days in crop which were significant in specific factors. The enviromental variance has a larger influence on the following characteres: width of leaves (LMFO), pulp weight, vitamin C contents (VITC), RMP20FR and growth of branches. The proportional large genetic variance components in CMFO, LMFO, plant height, treetop diameter, height of fruits, VITC and fruit weight make effective the genotype selection.
\end{abstract}

Index terms: genotypic variance, environmental variance, Malpighia emarginata.

\section{INTRODUÇÃO}

O estudo da variância e de seus componentes é útil para conhecimento e exploração das magnitudes dos caracteres no melhoramento da cultura. Assim, Vencovsky (1987), in Paterniani \& Viégas (1987), afirmou que, na variação, reside a viabilidade e o sucesso do trabalho de seleção no melhoramento das culturas. Em acréscimo, Ramalho et al. (1993) relataram que a variância é preferível à média porque esta última pode não representar com fidelidade o que ocorre na variável.

Segundo Brewbaker (1965), Johannsen foi o primeiro geneticista a propor divisão do valor fenotípico em componentes genéticos e ambientais e a afirmar que, além destes, a interação entre o genótipo e o ambiente pode ser considerada como um terceiro elemento.

Para Pinto (1995), a planta apresenta-se vigorosa por possuir bom patrimônio genético e/ou estar em situação nutricional privilegiada como pode demonstrar deficiências fenotípicas por possuir carga genética e/ou estar em ambiente desfavorável. Assim, faz-se importante a análise dos componentes da variância durante a avaliação fenotípica, dissociando-se o mérito genético da resposta ambiental.

Fisher, desde 1918, utilizou a variância nas metodologias estatístico-genéticas para obtenção dos componentes da variância genética, seguido por Allard (1971), Falconer (1981), Hallauer \& Miranda Filho (1982), Vencovsky (1987) in Paterniani \& Viégas (1987). Os componentes da variância são definidos, entre outros fatores, em função do delineamento adotado, da densidade de plantio e das épocas de avaliação.

Com as estimativas de componentes da variância, é possível compreender como cada caráter responde às condições ambientais, a fatores de variação e o quanto se deve à variação genética. A participação porcentual de cada componente na variação total de um caráter possibilita interpretação objetiva do

1 (Trabalho 110/2001). Recebido: 21/05/2001. Aceito para publicação: 22/02/2002. Extraído da tese de Doutoramento do primeiro autor desenvolvida na FCAV-UNESP. Financiada pela Fundação de Amparo à Pesquisa do Estado de São Paulo (FAPESP).

2 Biólogo Doutor em Agronomia, Dep. Ciências Exatas da FCAV-UNESP, Via de acesso Prof. Paulo Donato Castellane, s/n, CEP 14.884-900, Jaboticabal, SP.

3 Eng ${ }^{\circ}$ Agr ${ }^{\circ}$, Prof. Titular, Dep. de Ciências Exatas da FCAV-UNESP, Bolsista do CNPq, Via de acesso Prof. Paulo Donato Castellane, s/n, CEP 14.884-900, Jaboticabal, SP. Email: perecin@ fcav.unesp.br.

4 Eng ${ }^{-}$Agro ${ }^{\circ}$, Prof. Assistente Dr., Dep. de Produção Vegetal da FCAV-UNESP, Via de acesso Prof. Paulo Donato Castellane, s/n, CEP 14.884-900, Jaboticabal, SP. Email: baldo@fcav.unesp.br. 
que deve ser considerado para o melhoramento.

Objetivou-se estimar componentes da variância e sua participação porcentual na variação total nos caracteres dos genótipos de acerola de Itápolis, Viradouro e Jaboticabal.

\section{MATERIAL E MÉTODOS}

Os experimentos para estimar os componentes da variância nos aspectos vegetativos e produtivos foram executados em três localidades do Estado de São Paulo: Itápolis, Viradouro e Jaboticabal.

Em Itápolis, a $21^{\circ} 35^{\prime}$ de latitude sul, $48^{\circ} 47^{\prime}$ de longitude oeste e altitude de $560 \mathrm{~m}$ (Dicionário Geográfico Brasileiro, 1970), o experimento ocorreu em solo podzolizado Lins-Marília não irrigado, com plantas de seis anos e grande variabilidade, das quais, entre as melhores, selecionaram-se, mediante produção, arquitetura da árvore e tamanho dos frutos, 12 genótipos, numerados de I1 a I12 .

Em Viradouro, a $22^{\circ} 40$ ' de latitude sul, $48^{\circ} 40^{\prime}$ de longitude oeste e a $777 \mathrm{~m}$ de altitude, de pomar em latossolo roxo não irrigado, com aproximadamente cinco anos, foram selecionados, seguindo-se os mesmos critérios de Itápolis, dezoito genótipos, numerados de V1 a V18.

Jaboticabal, a $21^{\circ} 16^{\prime}$ de latitude sul e $48^{\circ} 19^{\prime}$ de longitude oeste com altitude de $575 \mathrm{~m}$, apresenta, segundo Köppen, clima tipo Cwa, precipitação anual de $1400 \mathrm{~mm}$, registrando, no mês mais seco, 13,9 mm e insolação de 2.259,1 horas/ano; da Faculdade de Ciências Agrárias e Veterinárias (FCAV), foram analisados onze genótipos com idade entre seis e vinte anos, numerados de CL1 a CL11 e mais quatro clones (genótipos com duas plantas cada, propagadas por estacas), três denominados R1, R2, R3 e o outro, P4, é o Florida Sweet, importado.

As avaliações foram realizadas nos caracteres de folhas, frutos e em outros das plantas, nos períodos de dez/97 a mar/98, em Itápolis; de jan/98 a jan/99, em Viradouro; de dez/97 a jan/99, em Jaboticabal. Em folhas, avaliaram-se comprimento e largura; em frutos, altura, diâmetro, massa, massa de polpa, massa de vinte frutos, massa de polpa de vinte frutos, massa média em três amostras de vinte frutos, massa média de polpa em três amostras de vinte frutos, rendimento de polpa em três amostras de vinte frutos, teor de vitamina $\mathrm{C} \mathrm{em} 100 \mathrm{ml}$ de suco, pelo método de redução do iodo (Lara et al., 1976); pH, pelo método potenciométrico (Lara et al., 1976), sólidos solúveis totais ( ${ }^{\circ}$ BRIX), através de leitura refratométrica de Pearson (1973); dias em floração e dias em colheita; na planta, altura, diâmetro de copa, crescimento médio de ramos e crescimento total de vinte ramos por estádio de crescimento.

As análises de variância foram realizadas no SAS (SAS/ STAT, 1995), modelo com genótipos e épocas, sem interação, exceto para os clones com duas plantas cada, quando se adotou modelo com clones, épocas e clones x épocas. Utilizou-se o PROC VARCOMP (método MINVQ0) para estimar os componentes da variância, supondo todos os efeitos aleatórios, segundo os esquemas:

a) Análise em uma só época, em um ambiente.

\begin{tabular}{cc}
\hline Fontes de variação & Esperança do quadrado médio E (QM) \\
\hline Clones & $\sigma_{\text {P:C }}^{2}+n_{\mathrm{P}} \sigma_{C}^{2}$ \\
Plantas dentro de clones & $\sigma_{\text {P:C }}^{2}$ \\
\hline
\end{tabular}

$\mathrm{n}_{\mathrm{p}}$, número de plantas por clones; $\sigma_{\mathrm{P}: \mathrm{C}}^{2}$, variância de plantas dentro de clones (ambiente); $\sigma_{C}^{2}$, variância de clones (genética) b) Análise em várias épocas (ambiente médio de um local em várias épocas).

\begin{tabular}{|c|c|}
\hline Fontes de variação & Esperança do quadrado médio $\mathrm{E}(\mathrm{QM})$ \\
\hline $\begin{array}{c}\text { Clones } \\
\text { Plantas dentro de clones } \\
\end{array}$ & $\begin{array}{c}\sigma_{\mathrm{EP}: \mathrm{C}}^{2}+\mathrm{n}_{\mathrm{e}} \sigma_{\mathrm{P}: \mathrm{C}}^{2}+\mathrm{n}_{\mathrm{P}} \sigma_{\mathrm{EXC}}^{2}+\mathrm{n}_{\mathrm{e}} \mathrm{n}_{\mathrm{P}} \sigma_{\mathrm{C}}^{2} \\
\sigma_{\mathrm{EP}: \mathrm{C}}^{2}+\mathrm{n}_{\mathrm{e}} \sigma_{\mathrm{P}: \mathrm{C}}^{2} \\
\end{array}$ \\
\hline $\begin{array}{c}\text { Épocas } \\
\text { Épocas x Clones } \\
\text { Épocas x Plantas dentro de clones }\end{array}$ & $\begin{array}{c}\sigma_{E P: C}^{2}+n_{P} \sigma_{E C}^{2}+n_{C} n_{P} \sigma_{E}^{2} \\
\sigma_{E P: C}^{2}+n_{P} \sigma_{E C}^{2} \\
\sigma_{E P: C}^{2}\end{array}$ \\
\hline
\end{tabular}

Como os quatro clones com duas plantas cada não foram escolhidos de forma essencialmente aleatória e estavam em apenas um ambiente (Jaboticabal), adotou-se um modelo mais simples que o descrito no item anterior (b), segundo esquema:

\begin{tabular}{|c|c|}
\hline Fontes de variação & Esperança do quadrado médio E (QM) \\
\hline Clones & $\sigma^{2}{ }_{a}+n_{P} \sigma_{E C}^{2}+n_{P} n_{E} \sigma_{C}^{2}$ \\
\hline Épocas & $\sigma^{2}+n_{P} \sigma_{E C}^{2}+n_{P} n_{C} \sigma_{E}^{2}$ \\
\hline Clones x Épocas & $\sigma^{2}+n_{P} \sigma_{E C}^{2}$ \\
\hline Erro & $\sigma_{\mathrm{a}}^{2}$ \\
\hline
\end{tabular}

$\mathrm{n}_{\mathrm{P}}$, número de plantas por clone; $\mathrm{n}_{\mathrm{C}}$, número de clones; $\mathrm{n}_{\mathrm{E}}$, número de épocas; $\sigma^{2}$, variância de ambiente; $\stackrel{C}{ }^{2}$ EC , variância da interação épocas $x$ clones; $\sigma_{\mathrm{E}}^{2}$, variância de épocas; $\sigma_{\mathrm{C}}^{2}$, variância de clones.

Aproximação similar, tomando a interação época $\mathrm{x}$ genótipo como resíduo ou erro, foi usada quando não existiam repetições de plantas, o que aconteceu nas três localidades, ou seja:

\begin{tabular}{cc}
\hline Fontes de variação & Esperança do quadrado médio E (QM) \\
\hline Genótipos & $\sigma_{\mathrm{EC}^{2}+\mathrm{n}_{\mathrm{e}} \sigma_{\mathrm{C}}^{2}}$ \\
Épocas & $\sigma_{\mathrm{EC}}^{2}+\mathrm{n}_{\mathrm{c}} \sigma_{\mathrm{E}}^{2}$ \\
Erro & $\sigma_{\mathrm{EC}}$ \\
\hline
\end{tabular}

$\mathrm{n}_{\mathrm{C}}$, número de genótipos; $\mathrm{n}_{\mathrm{e}}$, número de épocas; $\sigma_{\mathrm{EC}}^{2}$, variância da interação épocas x clones; $\sigma_{E}^{2}$, variância de épocas; $\sigma_{C}^{2}$, variância de genótipos.

Para comparação dos componentes da variância dos caracteres, foi estimada a participação porcentual de cada um deles em relação à variância total ou soma dos componentes da variância.

\section{RESULTADOS E DISCUSSÃO}

Na Tabela 1, encontram-se os resultados das análises de variância dos caracteres estudados. Para facilitar, a discussão efetuar-se-á em duas partes.

Clones em Jaboticabal com duas plantas cada um.

As duas plantas obtidas por propagação vegetativa de cada clone permitiram avaliar a interação clone x época. Como elas se localizam em ambiente restrito, o quadrado médio do erro da análise foi tomado como referência do mínimo de variância ambiental em caracteres de acerola. É interessante notar, entretanto, que algumas características de folhas e de altura de planta apresentam quadrado médio do erro menores que nos outros locais, possivelmente pela interação não significativa com épocas, refletindo variações casuais.

Verificam-se diferenças significativas em clones, épocas e clones x épocas nos caracteres altura média de fruto (TMFR), diâmetro médio de fruto (LMFR), massa de vinte frutos (PE20), medida de sólidos solúveis totais, massa média de vinte frutos (PM20FR), massa média de polpa (PMP20FR), ambas obtidas em três amostras de vinte frutos; crescimento total em vinte ramos (CRT20R), crescimento médio de ramos (CRM) e dias em floração 
(DFLO). Notam-se interações significativas cujos quadrados médios são cinco vezes maiores, ou até mais, do que o quadrado médio do erro, fato mais particularmente presente nas características dos frutos, o que sugere caracteres fortemente dependentes de épocas.

Poucos são os caracteres que apresentam diferenças significativas em apenas uma das fontes. Um caso, comprimento médio de folhas, deve-se ao clone R1, que possui folhas curtas e estreitas, diferentemente dos demais, o que não é influenciado por épocas (Tabela 1).

O rendimento médio de polpa em três amostras de vinte frutos apresenta diferenças significativas em épocas e na interação clones x épocas (Tabela 1), ocorrência justificada pela influência das condições meteorológicas, principalmente precipitação e temperatura, de acordo com Aróstegui et al. (1955). O caráter dias em colheita (DC) apresenta diferenças significativas em clones e épocas, fato atribuído ao vínculo que existe entre número de dias que a planta permanece em colheita com as condições meteorológicas de cada época e o tempo de colheita, que difere nos clones.

Largura de folha, altura de planta (ALT), diâmetro de copa (DICOP), massa média de fruto (PMFR), massa de polpa de vinte frutos (PP20) e pH apresentam diferenças significativas em clones e épocas, indicando que ocorrem diferenças entre clones com efeitos de épocas similares a todos.

Os coeficientes de variação para CMFO $(8,45)$, LMFO (9,14), ALT (8,34), TMFR (4,69), LMFR (3,57), PE20 (9,21), PMFR $(9,25), \mathrm{pH}(5,36),{ }^{\circ} \mathrm{BRIX}(9,42), \operatorname{PM} 20 \mathrm{FR}(9,96), \operatorname{DFLO}(6,00)$ e DC $(4,00)$ apresentaram-se baixos; em DICOP $(10,40)$, VITC $(16,97)$, PMP20FR (17,5) e RMP20FR (11,1), médios e em PP20 (24,01), PMPFR (23,76), CRT20R (38,00) e CRM (38,00), muito altos (Tabela 1), mesmo no ambiente restrito a Jaboticabal.

Na Tabela 2, observam-se os componentes da variância e sua participação porcentual na variância total. Constata-se que os caracteres CMFO, ALT, DICOP, RMP20FR e DC apresentam resultados negativos, provavelmente efeitos nulos, que ocasionalmente assim se apresentam em função do método utilizado na estimação.

Quanto à participação do componente variância ambiental nos diversos caracteres (Tabela 2), nota-se que um dado caráter, dependendo da sua natureza, pode sofrer maior influência ambiental do que outro. Os índices de LMFO $(44,12 \%)$, PP20 (33,62\%), VITC (33,02\%) e RMP20FR (35,15\%) demonstram que as condições ambientais interferem consideravelmente nesses caracteres. Em ALT (1,18\%), essa participação é muito baixa, uma vez que adquirido determinado porte, a planta poderá, no máximo, perder as folhas em função de adversidades meteorológicas, sem jamais reduzir sua altura.

A análise da interação épocas x clones demonstra que ${ }^{\circ}$ BRIX $(34,72 \%)$, PM20FR $(33,0 \%)$ e RMP20FR $(39,13 \%)$ apresentam os maiores índices de participação, o que sugere grande interação do genótipo com as condições ambientais nesses caracteres, indicando que, por eles, não se podem selecionar genótipos de acerola em avaliações restritas a apenas uma ou duas épocas. Em adição, constata-se que, em VITC, a interação épocas x clones é nula; no entanto, o componente de variação de épocas apresenta índice marcante, $30,34 \%$, indicando resposta diferenciada nas épocas.

Considerando o componente de variância genotípica ou de clones, observa-se que os índices de CMFO $(60,24 \%)$, ALT $(98,31 \%)$, TMFR $(43,91 \%)$ e VITC $(36,64 \%)$ são elevados; portanto, além de serem importantes no processo seletivo de genótipos de acerola, esses caracteres apresentam, nesta população, considerável variância genética que possibilita, a partir deles, ganho na seleção.

\section{repetição da planta.}

Genótipos de Itápolis, Viradouro e Jaboticabal, sem

Nestas análises, o quadrado médio do erro engloba os efeitos do ambiente e da interação genótipo x época. As diferenças genotípicas em cada localidade também são

TABELA 1 - Resultados da análise da variância dos caracteres em clones (duas plantas cada) e genótipos (uma planta cada) de acerola de Itápolis, Viradouro e Jaboticabal, nos períodos de dezembro/97 a março/98, janeiro/98 a janeiro/99 e dezembro/97 a janeiro/99, respectivamente.

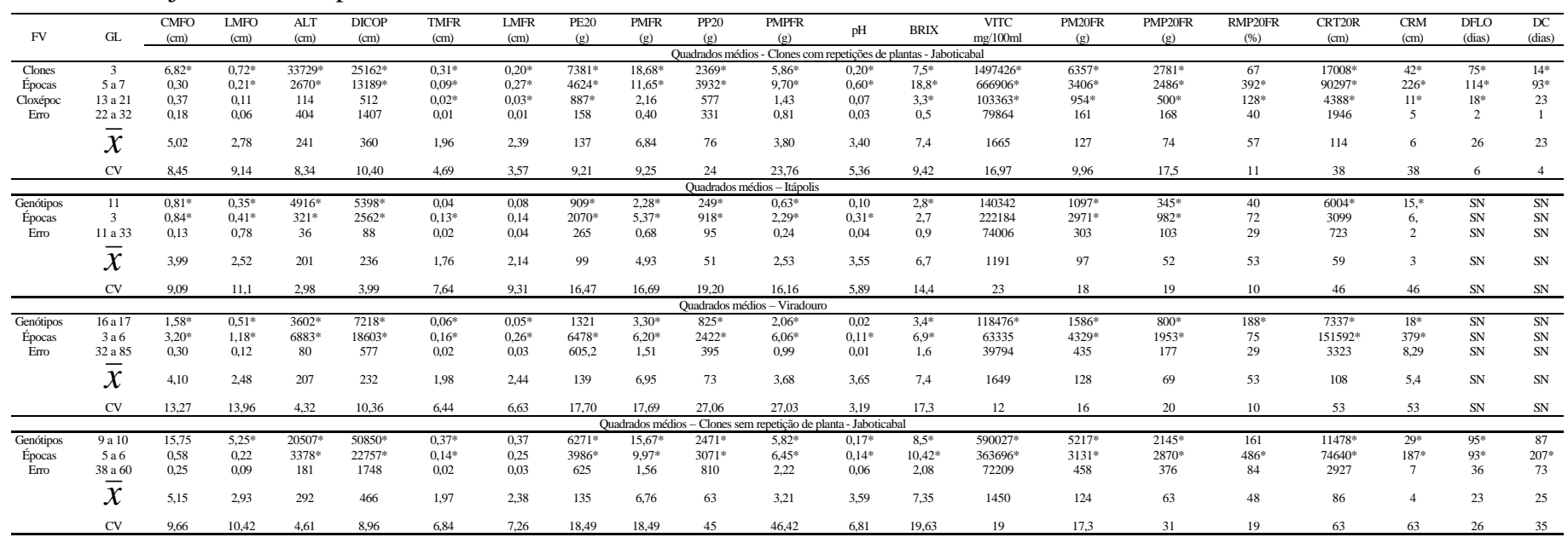

1CMFO, comprimento médio de folhas; LMFO, largura média de folhas; ALT, altura de planta; DICOP, diâmetro de copa; TMFR, altura média de fruto; LMFR, diâmetro médio de fruto; PE20, massa de vinte frutos; PMFR, massa média de fruto; PP20, massa de polpa de vinte frutos; PMPFR, massa média de polpa em fruto; $\mathrm{pH}$, medida de $\mathrm{pH}$; BRIX, medida de sólidos solúveis ; VITC, quantidade de vitamina C em $100 \mathrm{ml}$ de suco; PM20FR, massa média de vinte frutos; PMP20FR, massa média de polpa de vinte frutos; RMP20FR, rendimento médio de polpa de vinte frutos; CRT20R, crescimento total em vinte ramos; CRM, crescimento médio de ramos; DFLO, dias em floração; DC, dias em colheita; FV, fonte de variação; GL, graus de liberdade; Clo, clones; Époc, épocas; *significativo a $5 \%$ de probabilidade, pelo teste F; SN, sem observação; $\bar{x}$, média ; CV, coeficiente de variação. 
TABELA 2 - Estimativas dos componentes da variância nos caracteres e participação porcentual dos componentes na variância total nos clones de acerola, com repetições de plantas de dezembro/97 a janeiro/99, em Jaboticabal-SP.

\begin{tabular}{|c|c|c|c|c|c|c|c|c|c|c|c|c|c|c|c|c|c|c|c|c|}
\hline CPV & \multicolumn{20}{|c|}{ Caracteres estudados } \\
\hline $\begin{array}{c}\text { E } \\
\text { PPVT }\end{array}$ & $\begin{array}{c}\text { CMFO } \\
(\mathrm{cm})\end{array}$ & $\begin{array}{c}\text { LMFO } \\
(\mathrm{cm})\end{array}$ & $\begin{array}{l}\text { ALT } \\
(\mathrm{cm})\end{array}$ & $\begin{array}{c}\text { DICOP } \\
(\mathrm{cm})\end{array}$ & $\begin{array}{l}\text { TMFR } \\
(\mathrm{cm})\end{array}$ & $\begin{array}{l}\text { LMFR } \\
(\mathrm{cm})\end{array}$ & $\begin{array}{c}\text { PE20 } \\
\text { (g) }\end{array}$ & $\begin{array}{c}\text { PMFR } \\
\text { (g) }\end{array}$ & $\begin{array}{c}\text { PP20 } \\
\text { (g) }\end{array}$ & $\begin{array}{c}\text { PMPFR } \\
(\mathrm{g})\end{array}$ & $\mathrm{pH}$ & BRIX & $\begin{array}{c}\text { VITC } \\
\mathrm{mg} / 100 \mathrm{ml}\end{array}$ & $\begin{array}{l}\mathrm{PM} 20 \mathrm{FR} \\
\text { (g) }\end{array}$ & $\begin{array}{l}\text { PMP20FR } \\
\text { (g) }\end{array}$ & $\begin{array}{c}\text { RMP20FR } \\
(\%)\end{array}$ & $\begin{array}{c}\text { CRT20R } \\
(\mathrm{cm})\end{array}$ & $\begin{array}{l}\text { CRM } \\
(\mathrm{cm})\end{array}$ & $\begin{array}{l}\text { DFLO } \\
\text { (dias) }\end{array}$ & $\begin{array}{c}\mathrm{DC} \\
\text { (dias) }\end{array}$ \\
\hline$\sigma_{a}^{2}$ & 0,180 & 0,060 & 403,65 & 1407,3 & 0,008 & 0,007 & 158,3 & 0,40 & 331,4 & 0,81 & 0,030 & 0,48 & 79864,2 & 161,0 & 167,98 & 39,7 & 1946,3 & 4,82 & 2,41 & 0,75 \\
\hline$\sigma^{2} \mathrm{EC}$ & 0,095 & 0,025 & $-144,88$ & $-447,6$ & 0,006 & 0,012 & 364,36 & 0,88 & 123,0 & 0,31 & 0,020 & 1,42 & 0,65 & 396,6 & 165,96 & 44,2 & 1220,7 & 3,06 & 7,99 & 11,08 \\
\hline$\sigma^{2} \mathrm{E}$ & $-0,009$ & 0,013 & 319,48 & 1589,69 & 0,009 & 0,030 & 467,09 & 1,19 & 419,4 & 1,03 & 0,063 & 1,93 & 73380,0 & 306,5 & 248,20 & 32,9 & 10738,6 & 27,04 & 11,98 & 8,71 \\
\hline$\sigma^{2} \mathrm{C}$ & 0,403 & 0,038 & 33615,80 & 1540,65 & 0,018 & 0,011 & 405,88 & 1,03 & 111,9 & 0,28 & 0,008 & 0,26 & 88597,5 & 337,7 & 142,55 & $-3,84$ & 788,7 & 1,96 & 3,52 & $-0,59$ \\
\hline$\sigma^{2} \mathrm{~T}$ & 0,669 & 0,136 & 34193,25 & 4090,04 & 0,041 & 0,060 & 1395,63 & 3,50 & 985,7 & 2,43 & 0,121 & 4,09 & 241842,35 & 1201,8 & 724,69 & 112,96 & 14694,3 & 36,88 & 25,90 & 19,95 \\
\hline $\mathrm{PP}^{2}{ }_{\mathrm{a}}$ & 26,90 & 44,12 & 1,18 & 34,41 & 19,51 & 11,67 & 11,34 & 11,43 & 33,62 & 33,33 & 24,79 & 11,74 & 33,02 & 13,40 & 23,18 & 35,15 & 13,25 & 13,07 & 9,31 & 3,76 \\
\hline $\mathrm{PP} \sigma^{2} \mathrm{EC}$ & 14,20 & 18,38 & $-0,42$ & $-10,90$ & 14,63 & 20,00 & 26,11 & 25,14 & 12,48 & 12,76 & 16,53 & 34,72 & 0,00 & 33,00 & 22,90 & 39,13 & 8,31 & 8,30 & 30,85 & 55,54 \\
\hline $\mathrm{PP}^{2} \mathrm{E}$ & $-1,34$ & 9,56 & 0,93 & 38,86 & 21,95 & 50,00 & 33,47 & 34,00 & 42,55 & 42,39 & 52,07 & 47,19 & 30,34 & 25,50 & 34,25 & 29,13 & 73,08 & 73,31 & 46,25 & 43,66 \\
\hline $\mathrm{PP}^{2} \mathrm{C}$ & 60,24 & 27,94 & 98,31 & 37,67 & 43,91 & 18,33 & 29,08 & 29,43 & 11,35 & 11,52 & 6,61 & 6,35 & 36,64 & 28,10 & 19,67 & $-3,41$ & 5,36 & 5,32 & 13,59 & $-2,96$ \\
\hline
\end{tabular}

$\overline{\mathrm{CPV}}$, componentes da variância; PPVT, participação porcentual dos componentes na variância total; $\sigma^{2} \mathrm{a}$, variância ambiental; $\sigma^{2} \mathrm{EC}$, variância da interação épocas x clones; $\sigma^{2} \mathrm{E}$, variância de épocas; $\sigma^{2} \mathrm{C}$, variância de clones; $\sigma^{2} \mathrm{~T}$, variância total; $\mathrm{PP} \sigma^{2} \mathrm{a}$, participação porcentual da variância ambiental; PP $\sigma^{2} \mathrm{EC}$, participação porcentual da variância da interação épocas x clones; PP $\sigma^{2} E$, participação porcentual da variância de épocas; PPo ${ }^{2} \mathrm{C}$, participação porcentual da variância de clones; CMFO, comprimento médio de folhas; LMFO, largura média de folhas; ALT, altura de planta; DICOP, diâmetro de copa; TMFR, altura média de fruto; LMFR, diâmetro médio de fruto; PE20, massa de vinte frutos; PMFR, massa média de fruto; PP20, massa de polpa de vinte frutos; PMPFR, massa média de polpa em fruto; $\mathrm{pH}$, medida de $\mathrm{pH}$; BRIX, medida de sólidos solúveis; VITC, quantidade de vitamina C em 100 ml de suco; PM20FR, massa média de vinte frutos; PMP20FR, massa média de polpa de vinte frutos; RMP20FR, rendimento médio de polpa de vinte frutos; CRT20R, crescimento total em vinte ramos; CRM, crescimento médio de ramos; DFLO, dias em floração; DC, dias em colheita.

TABELA 3 - Estimativas dos componentes da variância nos caracteres e participação porcentual dos componentes na variância total, nos genótipos de acerola, sem repetição de planta, de Itápolis, Viradouro e Jaboticabal, período de dezembro/97 a março/98, janeiro/98 a janeiro/99 e dezembro/97 a janeiro/99, respectivamente.

\begin{tabular}{|c|c|c|c|c|c|c|c|c|c|c|c|c|c|c|c|c|c|c|c|}
\hline \multicolumn{20}{|c|}{ Caracteres estudados } \\
\hline $\begin{array}{l}\overline{\text { MFO }} \\
\mathrm{cm})\end{array}$ & $\begin{array}{c}\text { LMFO } \\
(\mathrm{cm})\end{array}$ & $\begin{array}{l}\text { ALT } \\
(\mathrm{cm})\end{array}$ & $\begin{array}{l}\text { DICOP } \\
(\mathrm{cm})\end{array}$ & $\begin{array}{l}\text { TMFR } \\
(\mathrm{cm})\end{array}$ & $\begin{array}{l}\text { LMFR } \\
(\mathrm{cm})\end{array}$ & $\begin{array}{c}\text { PE20 } \\
(\mathrm{g})\end{array}$ & $\begin{array}{c}\text { PMFR } \\
(\mathrm{g})\end{array}$ & $\begin{array}{c}\text { PP20 } \\
\text { (g) }\end{array}$ & $\begin{array}{l}\text { PMPFR } \\
\text { (g) }\end{array}$ & $\mathrm{pH}$ & BRIX & $\begin{array}{c}\text { VITC } \\
\mathrm{mg} / 100 \mathrm{ml}\end{array}$ & $\begin{array}{l}\text { PM20FR } \\
\text { (g) }\end{array}$ & $\begin{array}{c}\text { PMP20FR } \\
\text { (g) }\end{array}$ & $\begin{array}{c}\text { RMP20FR } \\
\text { (\%) }\end{array}$ & $\begin{array}{c}\text { CRT20R } \\
(\mathrm{cm})\end{array}$ & $\begin{array}{l}\text { CRM } \\
(\mathrm{cm})\end{array}$ & $\begin{array}{l}\text { DFLO } \\
\text { (dias) }\end{array}$ & $\begin{array}{c}\text { DC } \\
\text { (dias) }\end{array}$ \\
\hline \multicolumn{20}{|c|}{ Itápolis } \\
\hline 1322 & 0,0783 & 36,13 & 88,32 & 0,0182 & 0,0396 & 264,4 & 0,68 & 94,51 & 0,24 & 0,044 & 0,928 & 74006,3 & 302,79 & 102,77 & 29,05 & 723,08 & 1,72 & SN & SN \\
\hline 0591 & 0,0279 & 23,77 & 206,12 & 0,0097 & 0,0083 & 150,5 & 0,39 & 68,60 & 0,17 & 0,022 & 0,144 & 12348,2 & 222,38 & 73,29 & 3,55 & 197,96 & 0,36 & $\mathrm{SN}$ & $\mathrm{SN}$ \\
\hline 1693 & 0,0670 & 1220,07 & 1327,50 & 0,0061 & 0,0104 & 161,0 & 0,40 & 38,70 & 0,097 & 0,0155 & 0,478 & 16583,9 & 198,49 & 60,56 & 2,80 & 1320,29 & 3,42 & SN & SN \\
\hline 3606 & 0,1732 & 1279,97 & 1621,94 & 0,034 & 0,0583 & 576,0 & 1,47 & 201,81 & 0,507 & 0,0815 & 1,55 & 102938,4 & 723,66 & 236,62 & 35,4 & 2241,33 & 5,50 & SN & SN \\
\hline 6,66 & 45,21 & 2,82 & 5,45 & 53,53 & 67,92 & 45,90 & 46,26 & 46,83 & 47,34 & 53,99 & 59,87 & 71,89 & 41,84 & 43,43 & 82,06 & 32,26 & 31,27 & SN & SN \\
\hline 6,39 & 16,11 & 1,86 & 12,70 & 28,53 & 14,24 & 26,14 & 26,53 & 33,99 & 33,53 & 26,99 & 9,29 & 11,99 & 30,73 & 30,98 & 10,03 & 8,83 & 6,55 & SN & SN \\
\hline 6,95 & 38,68 & 95,32 & 81,85 & 17,94 & 17,84 & 27,96 & 27,21 & 19,18 & 19,13 & 19,02 & 30,84 & 16,12 & 27,43 & 25,59 & 7,91 & 58,91 & 62,18 & SN & SN \\
\hline \multicolumn{20}{|c|}{ Viradouro } \\
\hline$\overline{\mathrm{j}, 30}$ & 0,12 & 79,75 & 577,40 & 0,016 & 0,030 & 605,2 & 1,51 & 395,42 & 0,99 & 0,01 & 1,63 & 39793,5 & 435,17 & 176,88 & 28,80 & 3323,32 & 8,29 & SN & SN \\
\hline ),16 & 0,059 & 377,94 & 1001,40 & 0,008 & 0,018 & 326,2 & 0,26 & 112,59 & 0,28 & 0,006 & 0,297 & 1307,9 & 216,30 & 98,67 & 2,57 & 8237,2 & 20,59 & $\mathrm{SN}$ & $\mathrm{SN}$ \\
\hline , 18 & 0,056 & 503,24 & 948,69 & 0,008 & 0,003 & 102,2 & 0,26 & 61,43 & 0,153 & 0,0014 & 0,247 & 11240,41 & 164,46 & 89,04 & 22,67 & 573,3 & 1,43 & SN & $\mathrm{SN}$ \\
\hline ),64 & 0,235 & 960,93 & 2527,49 & 0,032 & 0,051 & 1033 , & 2,03 & 569,44 & 1,423 & 0,0174 & 2,174 & 52341,81 & 815,93 & 364,59 & 54,04 & 12133,82 & 30,31 & SN & SN \\
\hline 6,87 & 51,06 & 8,30 & 22,85 & 50,00 & 58,82 & 58,54 & 74,38 & 69,44 & 69,57 & 57,47 & 74,98 & 76,03 & 53,33 & 48,52 & 53,29 & 27,39 & 27,35 & SN & SN \\
\hline 25,0 & 25,11 & 39,33 & 39,62 & 25,00 & 35,29 & 31,56 & 12,81 & 19,77 & 19,68 & 34,48 & 13,66 & 2,50 & 26,51 & 27,06 & 4,76 & 67,89 & 67,93 & SN & SN \\
\hline \multirow{2}{*}{\multicolumn{20}{|c|}{ Jaboticabal }} \\
\hline & & & & & & & & & & & & & & & & & & & \\
\hline$\overline{\mathrm{J}, 25}$ & 0,09 & 180,7 & 1748,3 & 0,02 & 0,03 & 624,9 & 1,56 & 810,12 & 2,22 & 0,06 & 2,08 & 72208,50 & 457,80 & 376,30 & 83,73 & 2926,65 & 7,31 & 36,22 & 72,81 \\
\hline, 03 & 0,012 & 290,64 & 1909,9 & 0,011 & 0,02 & 305,5 & 0,76 & 205,49 & 0,38 & 0,007 & 0,76 & 26498,85 & 243,04 & 226,74 & 36,76 & 6519,39 & 16,29 & 5,17 & 12,15 \\
\hline 2,21 & 0,737 & 2903,7 & 7014,6 & 0,05 & 0,048 & 806,5 & 2,02 & 237,32 & 0,52 & 0,016 & 0,92 & 73974,08 & 679,80 & 252,60 & 11,02 & 1221,58 & 3,08 & 8,33 & 2,09 \\
\hline 2,49 & 0,839 & 3375,04 & 10672,8 & 0,081 & 0,098 & 1737 , & 4,34 & 1252,93 & 3,12 & 0,083 & 3,76 & 172681,43 & 1380,64 & 855,64 & 131,51 & 10667,62 & 26,68 & 49,72 & 87,05 \\
\hline 0,04 & 10,73 & 5,35 & 16,38 & 24,69 & 30,61 & 35,98 & 35,94 & 64,66 & 71,15 & 72,30 & 55,32 & 41,82 & 33,16 & 43,98 & 63,67 & 27,43 & 27,40 & 72,84 & 83,64 \\
\hline 1,20 & 1,43 & 8,62 & 17,90 & 13,58 & 20,41 & 17,59 & 17,52 & 16,40 & 12,18 & 8,41 & 20,21 & 15,34 & 17,60 & 26,50 & 27,95 & 61,12 & 61,06 & 10,40 & 13,96 \\
\hline 8,76 & 87,84 & 86,03 & 65,72 & 61,73 & 48,98 & 46,43 & 46,54 & 18,94 & 16,67 & 19,29 & 24,47 & 42,84 & 49,24 & 29,52 & 8,38 & 11,45 & 11,54 & 16,76 & 2,40 \\
\hline
\end{tabular}

$\mathrm{CPV}$, componentes da variância; PPVT, participação porcentual dos componentes na variância total; $\sigma^{2} \mathrm{EC}$, variância da interação épocas x genótipos; $\sigma^{2} \mathrm{E}$, variância de épocas; $\sigma^{2} \mathrm{C}$, variância de genótipos; $\sigma^{2} \mathrm{~T}$, variância total; $\mathrm{PP} \sigma^{2} \mathrm{EC}$, participação porcentual da variância da interação épocas x genótipos; $P P \sigma^{2} \mathrm{E}$, participação porcentual da variância de épocas; $\mathrm{PP} \sigma^{2} \mathrm{C}$, participação porcentual da variância de genótipos; CMFO, comprimento médio de folhas; LMFO, largura média de folhas; ALT, altura de planta; DICOP, diâmetro de copa; TMFR, altura média de fruto; LMFR, diâmetro médio de fruto; PE20, massa de vinte frutos; PMFR, massa média de fruto; PP20, massa de polpa de vinte frutos; PMPFR, massa média de polpa em fruto; pH, medida de pH; BRIX, medida de sólidos solúveis; VITC, quantidade de vitamina C em $100 \mathrm{ml}$ de suco; PM20FR, massa média de vinte frutos; PMP20FR, massa média de polpa de vinte frutos; RMP20FR, rendimento médio de polpa de vinte frutos; CRT20R, crescimento total em vinte ramos; CRM, crescimento médio de ramos; DFLO, dias em floração; DC, dias em colheita; SN, sem observações.

responsáveis por parte das diferenças apresentadas na interação com épocas.

Na Tabela 1, encontram-se as análises de variância nos genótipos de Itápolis, Viradouro e Jaboticabal. Há diferenças significativas simultâneas em genótipos e épocas nos caracteres CMFO, LMFO, ALT, PE20, PMFR, PP20, PMPFR, PM20FR e PMP20FR, em Itápolis; em CMFO, LMFO, ALT, DICOP, TMFR, LMFR, PMFR, PP20, PMPFR, 'BRIX, PM20FR, PMP20FR, CRT20R e CRM, em Viradouro e em ALT, DICOP, TMFR, PE20, PMFR, PP20, PMPFR, pH, ${ }^{\circ}$ BRIX, VITC, PM20FR, PMP20FR, CRT20R, CRM e DFLO, em Jaboticabal. Alguns caracteres demonstram diferenças em cada localidade, principalmente se observados pH e VITC com diferenças significativas em genótipos e épocas, em Jaboticabal; em Itápolis, apenas o pH difere em época e, em Viradouro, o pH difere em época e a quantidade de vitamina $\mathrm{C}$ em genótipo (Tabela 1 ).

Alguns caracteres mostram diferenças significativas em apenas uma das fontes de variação, de maneira que, se for considerado o fator genótipo, ${ }^{\circ} \mathrm{BRIX}, \mathrm{CRT} 20 \mathrm{R}$ e CRM, diferem significativamente, em Itápolis; VITC, RMP20FR, em Viradouro e LMFO, em Jaboticabal. Considerando o fator época, TMFR e $\mathrm{pH}$ diferem significativamente, em Itápolis, PE20 e pH, em Viradouro, e RMP20FR, em Jaboticabal. Os dados sugerem ainda que os grupos genéticos apresentam, em alguns caracteres, respostas específicas e particularizadas em função da composição genotípica do grupo populacional e das épocas estudadas, que não foram coincidentes (Tabela 1).

Os caracteres LMFR, VITC, RMP20FR não apresentam 
diferenças significativas em nenhuma das fontes em Itápolis, nem CMFO, em Jaboticabal, câmpus (Tabela 1).

Os caracteres DFLO e DC foram estudados apenas em Jaboticabal (Tabela 1); não se verificam, em relação a DC, diferenças significativas em genótipo.

Nota-se certa proximidade dos valores absolutos dos coeficientes de variação da Tabela 1 nos caracteres CMFO,LMFO,TMFR, LMFR, pH, VITC, PM20FR e RMP20FR em todas as localidades, sugerindo influências similares de ambiente e da interação genótipos x época. Observam-se, ainda, pequenas diferenças nos valores dos coeficientes dos caracteres ALT, DICOP, PE20, PMFR, PP20, PMFR, ${ }^{\circ}$ BRIX, PMP20FR e RMP20FR, em todas as localidades, e DFLO e DC, nos dois grupos de Jaboticabal, evidenciando possíveis diferenças de genótipos e de sua interação com épocas. Diferenças na idade das plantas ocasionam CV mais elevado em relação a ALT e DICOP, em Jaboticabal (nos dois outros locais, as plantas tinham idades similares).

A comparação das Tabelas 1 e 2 demonstra que ${ }^{\circ}$ BRIX, PM20FR, PMP20FR e RMP20FR apresentam valores elevados para o quadrado médio do erro e o componente da variância de época x clones, reforçando que a seleção baseada em tais caracteres deve ser criteriosa, já que existe interação de genótipos $\mathrm{x}$ épocas, semelhantemente para DFLO e DC, avaliados em Jaboticabal.

O componente época interfere nas variações de TMFR, LMFR, PE20, PMFR, PP20, PMPFR, pH, ${ }^{\circ}$ BRIX, VITC, PM20FR,PMP20FR, RMP20FR (Tabelas 2 e 3). O mesmo ocorre com CRT20R e CRM, determinantes do componente da variância de épocas nos caracteres ALT e DICOP.

No contexto geral, destaca-se que CMFO, LMFO, ALT, DICOP, TMFR, PMFR e PE20 são importantes na seleção de genótipos por possuírem elevada participação dos componentes genéticos na variação total (Tabelas 2 e 3 ).

\section{CONCLUSÕES}

1. Os componentes da variância ambiental e de épocas interferem diferentemente nos caracteres, demonstrando efeito nas variações de largura média de folhas (LMFO), massa de polpa de vinte frutos (PP20), quantidade de vitamina C em $100 \mathrm{ml}$ de suco (VITC), rendimento médio de polpa de vinte frutos (RMP20FR) e crescimento médio de ramos (CRM)

2. O componente de variação de épocas x clones é o mais influente em medida de sólidos solúveis ( $\left.{ }^{\circ} \mathrm{BRIX}\right)$, massa média de vinte frutos (PM20FR), massa média de polpa de vinte frutos (PMP20FR) e RMP20FR; esses caracteres devem ser descartados como critério de seleção se as avaliações abrangerem apenas uma ou duas épocas.

3. O comprimento médio de folhas (CMFO), LMFO, altura de planta (ALT), diâmetro de copa (DICOP), altura média de fruto (TMFR), VITC, massa média de fruto (PMFR) e massa de vinte frutos (PE20) apresentam consideráveis variâncias devidas ao componente genético, possibilitando seleção.

\section{AGRADECIMENTOS}

À FAPESP, pela concessão de bolsa e apoio financeiro ao projeto.

\section{REFERÊNCIAS BIBLIOGRÁFICAS}

ALLARD, R.W. Princípios do melhoramento genético das plantas. São Paulo: Edgard Blucher, 1971.381p.

ARÓSTEGUI, F.; ASENJO, C.F.; MUNIZ, A.I.; ALEMANY, L.. Observations and data on a promissing selection of the West Indian Cherry, M. punicifolia L. Journal Agricultural, Mayaguez, v.39, n.2, 1955, p. 51-56.

BREWBAKER, J.L. Agricultural genetics. New Jersey: Prentics Hall, 1965. 156p.

DICIONÁRIO geográfico brasileiro. Porto Alegre: Globo, 1970. $559 \mathrm{p}$

FALCONER, D. S. Introdução à genética quantitativa. Viçosa: Universidade Federal de Viçosa, 1981. 279p.

HALLAUER, A.R.; MIRANDA FILHO, J.B. Quantitative genetics in maize breeding. Ames: Iowa State University Press, 1982. 486p.

LARA, A.B.W.; NAZÁRIO, G.; ALMEIDA, M.E.W.; PREGNOLATO, W. Normas analíticas do Instituto Adolfo Lutz - Métodos Químicos e Físicos para análises de alimentos. 2(Ed). São Paulo: Instituto Adolfo Lutz, 1976.

PEARSON, D. Laboratory techniques in food analysis. London: London Butterwoths, 1973. p 58-60.

PINTO, R.J.B. Introdução ao melhoramento genético de plantas. Maringá: Editora da Universidade Estadual de Maringá, 1995. $275 p$.

RAMALHO, M.A.P.; SANTOS, J.B. dos; ZIMMERMANN, M.J. de O. Genética quantitativa em plantas autógamas - aplicação ao melhoramento do feijoeiro. Editora UFG, 1993. 271p.

SAS/STAT. User Guide (Release 6.11). Cary, NC.: SAS Inst. 1995.

VENCOVSKY, R. Herança quantitativa. In: PATERNIANI, E.; VIÉGAS, G.P. Melhoramento e produção de milho. Campinas: Fundação Cargill, 1987. p. 137-209. 